\title{
ER Stress Activating ATF4/CHOP-TNF- $\alpha$ Signaling Pathway Contributes to Alcohol- Induced Disruption of Osteogenic Lineage of Multipotential Mesenchymal Stem Cell
}

\author{
Yueping Chen ${ }^{a, b}$ Hui Gao ${ }^{b}$ Qingshui Yin ${ }^{c}$ Liang Chen $^{b}$ Panfeng Dong ${ }^{b}$ \\ Xiaoyun Zhang ${ }^{\mathrm{b}}$ Jie Kang ${ }^{\mathrm{b}}$ \\ aSouthern Medical University, 2010 MD TongHe, Guangzhou; bepartment of Orthopedics, Affiliated \\ Rui Kang Hospital of Guangxi Traditional Chinese Medical School, Nanning 530011, Guangxi Zhuang \\ Autonomous Region; 'Orthopaedic Hospital, People's Liberation Army General Hospital of Guangzhou \\ Military Command, Guangzhou 510010, Guangdong
}

\section{Key Words}

Ethanol - Multipotential mesenchymal stem cells (MSC) - Bone-marrow stem cells (BMSCs) • Osteogenesis • Adipogenesis • Reactive oxygen species (ROS) • ER stress • Heat shock protein - ATF4 - CHOP • TNF- $\alpha$

\begin{abstract}
Background/Aims: Studies have provided substantial evidence that osteoblasts and adipocytes share common progenitor-multipotential mesenchymal stem cells in bone marrow (BMSCs), and excessive alcohol consumption shifts away from osteogenic to adipogenic lineage. However, how exactly alcohol impairs osteogenesis is still incompletely understood. This study was designed to shed light on this issue. Methods: We treated primary BMSCs from human subjects with alcohol for 24 days. We measured changes of genes related to endoplasmic reticulum (ER) stress, adipogenic markers and osteogenic markers using quantitative real-time RT-PCR and Western blot analysis. We performed Oil red $O$ staining and quantification of adipogenesis. We also conducted caspase 3 activity assay to assess BMSC apoptosis. Results: We showed here that chronic exposure of BMSCs to alcohol induced adipogenesis and disrupted osteogenesis as indicated by upregulation of adipogenic markers (PPARY2 and aP2), downregulation of osteogenic markers (Osf2/Cbfa1), and accumulation of lipid droplets. Alcohol induced ER stress, as reflected by increased expression of glucoseregulated proteins GRP78 and GRP94, and by increased expression of transcription factors activating transcription factor 4 (ATF4) and C/EBP homologous protein (CHOP), and enhanced caspase 3 activity. Additionally, ER stress also upregulated tumor necrosis factor-alpha (TNF- $\alpha$ ). Simultaneous silencing of ATF4 and CHOP prevented upregulation of TNF- $\alpha$. Knockdown of either ATF4 and CHOP or TNF- $\alpha$ by their siRNAs was able to reverse the ethanol-induced


adipogenesis. Conclusion: Our data therefore revealed a role of ER stress and ATF4/CHOP in the ethanol-induced inhibition of osteogenesis, and activation of TNF- $\alpha$ signaling by ATF4/ $\mathrm{CHOP}$ linking ER stress to adipogenic lineage in response to alcohol stimulation. This work should establish a new signaling pathway linking alcohol, ER stress, and TNF- $\alpha$ to loss of bone formation: Ethanol $\rightarrow$ ER stress $\uparrow \uparrow \uparrow \rightarrow$ ATF4 \& CHOP $\uparrow \uparrow \rightarrow$ TNF- $\alpha \uparrow \uparrow \uparrow \rightarrow$ Osteoblasts $\downarrow \downarrow \downarrow$.

Copyright (c) 2013 S. Karger AG, Basel

\section{Introduction}

Osteoporosis is characterized by low bone mass resulting from an imbalance between bone resorption by osteoclasts and bone formation by osteoblasts. Therefore, decreased bone formation by osteoblasts may lead to the development of osteoporosis, and thus rate of apoptosis of osteoporosis is responsible for the regulation of bone formation. In adults, the osteoblast, which is responsible for bone formation, is derived from multipotential mesenchymal stem cells (MSC) in bone marrow (BMSCs) [1-4]. Several studies have provided substantial evidence that osteoblasts and adipocytes share common progenitor: multipotential BMSCs, and the relationship between adipogenesis and osteogenesis is reciprocal with increasing adipogenesis accompanied by decreasing osteogenesis and vice versa.

After consumption, alcohol is readily distributed throughout the body in the blood stream and crosses biological membranes, affecting virtually all biological processes inside the cell. Excessive alcohol consumption substantially promotes adipogenesis and inhibits osteogenesis [5-9]. Moreover, both chronic and binge consumptions of ethanol are known to cause suppression of bone formation and enhancement of bone resorption. Thus, alcohol is considered a major risk factor for osteoporosis, a disease particularly prevalent in women worldwide [10]. Meanwhile, excessive alcohol exposure induces endoplasmic reticulum (ER) stress response, a condition under which unfolded/misfolded proteins accumulate in the ER [11-14]. ER stress contributes to alcoholic disorders of major organs such as liver, pancreas, heart, and brain. Multiple factors are involved in the complex process of ER stress including activating transcription factor 4 (ATF4) [15-17] and C/EBP homologous protein (CHOP), also known as growth arrest- and DNA damage-inducible gene 153 (GADD153) [1821], both of which can cause apoptotic cell death.

Intriguingly, ATF4 is an essential regulator of osteogenesis [22]. In osteoblasts, whose primary role in bone formation is building calcium enriched extracellular matrix (ECM), expression of ATF4 is coordinately regulated during the course of their differentiation [23]. ATF4 is also required for preserving mature osteoblast function including the synthesis of collagen, the most abundant extracellular protein found in bones [23-25]. It has been shown that a mutant mouse lacking the ATF4 gene is unable to form any part of a mature cytoskeleton [23]. ER stress leads to preferential translational expression by a mechanism involving phosphorylation of eukaryotic translation initiation factor- $2 \alpha$ (eIF $2 \alpha$ ) [15]. The phosphorylated eIF2 $\alpha$ in turn acts to activate the translation of ATF4. When ER stress is excessive, activation of ATF4 expression results in apoptosis. An intriguing question is the fate of osteoblasts that utilize ATF4 as a critical transcription factor during osteogenesis after excessive alcohol consumption.

Also notably, CHOP is another factor necessary for bone development and its anabolic effects have been reported to occur in response to bone morphogenetic protein [26-28], although overexpression of CHOP resulted in impaired bone formation. It has been shown that CHOP is expressed in bone as well as in mesenchymal progenitors and primary osteoblasts [27]. Overexpression of CHOP reduces alkaline phosphatase activity in primary osteoblasts and suppresses the formation of calcified bone nodules. CHOP-deficient osteoblasts differentiate more strongly than their wild-type counterparts, suggesting that endogenous CHOP plays an important role in the inhibition of osteoblast differentiation. Moreover, ATF4 can elevate expression of CHOP and both facilitate apoptosis $[18,19]$. A specific question on 
CHOP we wanted to address herein was similar to that on ATF4: how CHOP affects osteoblast lineage of BMSCs with excessive alcohol consumption.

Recently, it was documented that the ethanol-induced inhibition of osteogenesis is attenuated by administration of the antagonist to tumor necrosis factor (TNF)- $\alpha$, indicating that the osteoinhibitory effects of chronic ethanol exposure are mediated in part by the TNF- $\alpha$ signaling axis [29]. Indeed, it has been shown that in the bone marrow, the expression of TNF- $\alpha$ mRNA was significantly increased by ethanol [30]. However, it remained unclear how ethanol increases TNF- $\alpha$.

On the basis of the abovementioned facts, we proposed that alcohol exposure can trigger ER stress in BMSCs, which in turn causes aberrant overexpression of ATF4 and CHOP, leading to excessive activation TNF- $\alpha$ signaling pathway which in turn mediates inhibition of differentiation into osteoblasts thereby bone formation. The aim of the present study was to test this hypothesis.

\section{Materials and Methods}

Human BMSCs preparation and culture

Bone marrow samples for isolating BMSCs were collected from cancellous bony fragments of patients (age range $35-50$ years; $n=6$ ) undergoing routine total hip replacement surgery in the Orthopaedic Hospital, the People's Liberation Army General Hospital of Guangzhou Military Command. Informed written consent was obtained from all patients. The study protocol was approved by the Ethics Committee of the People's Liberation Army General Hospital of Guangzhou Military Command. None of the patients were alcoholics, showed any evidence of concurrent illness, and received any medications that could affect bone metabolism.

The procedures for preparing primary BMSCs from human subjects were essentially the same as described by Huang et al. [31], which were a modified method of Percoll density gradient centrifugation [32]. Briefly, when BMSC cultures became nearly confluent, cells were trypsinized and plated in $75 \mathrm{~cm}^{2}$ flasks or 6-well plates for protein analysis or RNA analysis and cytochemical analysis, respectively. Cells were used for siRNA transfection/adipogenic induction/control at passage two or three only. Cell surface markers were evaluated by flow cytometry (Cytomics FC-500, Beckman Coulter, CA, USA) using the monoclonal antibodies conjugated with fluorescein isothiocyanate (FITC) or phycoerythrine (PE): CD34FITC, CD105-PE, CD166-PE.

\section{Alcohol treatment and adipogenic induction}

BMSCs at passage two or three were divided into two groups: control group and alcohol group. The control group was cultured in Dulbecco's modified Eagle's medium (DMEM) with 10\% FBS and the alcohol group was cultured in DMEM containing 10\% FBS and $100 \mathrm{mM}$ alcohol for 24 days. This concentration of alcohol is approximately twice as much as its $\mathrm{IC}_{50}$ for inhibiting osteoblast proliferation in vitro and also represents a level within the physiological range observed in actively imbibing alcoholics [31,33]. To supplement the potential loss of alcohol because of its volatile nature and maintain the constant alcohol concentration, we followed the procedures described by Huang et al. [31] by placing a reservoir containing the same concentration of alcohol in a chamber during culture, which was replenished daily.

Small interfering RNA (siRNA)

The 21-nucleotide siRNA duplexes were designed and synthesized by Invitrogen. The siRNA sequences used here were as follows: CHOP sense: CUG AUU GAC CGA AUG GUG ATT; CHOP antisense: UCA CCA UUC GGU CAA UCA GTT [34]. ATF4 sense: 5'-CCA CGU UGG AUG ACA CUU GdTdT; ATF4 antisense: 5'-CAA GUG UCA UCC AAC GUG GdTdT [35]. Control sense: GAC UAC UGG UCG UUG AAC UTT. Control antisense: AGU UCA ACG ACC AGU AGU CTT. The transfection of siRNAs was performed with LipofectAMINE 2000 according to the manufacturer's instructions. Briefly, $16 \mu \mathrm{l}$ of LipofectAMINE 2000 reagent was mixed with $400 \mu \mathrm{l}$ of Opti-MEM (Invitrogen) at room temperature (RT) for $5 \mathrm{~min}$ and then incubated with a mixture of $12 \mu \mathrm{l}$ of 20 $\mu \mathrm{M}$ siRNA duplex and $400 \mu \mathrm{l}$ of Opti-MEM for an additional $20 \mathrm{~min}$ at RT. The complexes were then applied to cultured cells at $\sim 70 \%$ confluence on a $60-\mathrm{mm}$ plate containing $4 \mathrm{ml}$ of DMEM. After $12 \mathrm{~h}$ incubation, the medium was replaced with fresh DMEM supplemented with $10 \%$ fetal bovine serum and 1\% penicillin/ streptomycin. 
Caspase-3 activity assay

The caspase-3-like activity was measured by using a caspase-3 fluorometric assay kit (Sigma) according to the manufacturer's protocol. In brief, cellular extracts containing $50 \mu \mathrm{g}$ of total proteins were incubated in $200 \mu \mathrm{l}$ of assay buffer containing the synthetic fluorescent substrate Ac-DEVD-AMC at RT for 1 h. Fluorescence resulting from cleavage of the substrate was measured using a fluorometer with excitation at $380 \mathrm{~nm}$ and emission at $460 \mathrm{~nm}$.

\section{Oil red $O$ staining and quantification}

For morphological assessment of adipogenesis, the accumulation of intracellular lipid droplets was visualised by Oil red 0 staining. The cells were fixed in $4 \%$ neutral buffered formalin for ten min and washed with 3\% isopropanol, followed by incubation with a newly filtered Oil red 0 staining solution for $1 \mathrm{~h}$ at RT. After staining, the cells were rinsed with double-distilled $\mathrm{H}_{2} \mathrm{O}$. Cells were considered as lipid-positive when droplets were stained red under a light microscope. Lipid-positive cells were counted under 10 microscopic fields ( $\times 200$ magnification), with three fields per chamber slide to obtain an average number of lipidpositive cells for each group.

\section{Real-time PCR}

Total RNA was prepared from cultures on day ten, using the Trizol reagent (Invitrogen) according to the manufacturer's instructions. Quantitative real-time RT-PCR analysis was performed with the SYBR Green RT-PCR Kit (Qiagen, Valencia, CA) using the GeneAmp 5700 sequence detection system (Applied Biosystems) according to the manufacturer's instructions. Levels of mRNAs of adipogenic markers (PPAR 2 and aP2), osteogenic maker Osf2/Cbfa1, ATF4, CHOP, and TNF- $\alpha$ were determined. Samples were assayed in triplicates, and the values were normalized to the relative amounts of $\beta$-actin, and the results were interpreted using the $\Delta \mathrm{CT}$ method.

\section{Immunoblot analysis}

Cells were lysed in a RIPA lysis buffer containing inhibitors for proteases and phosphatases. Protein concentration was determined by the protein content was determined by BCA Protein Assay Kit using bovine serum albumin as the standard (Pierce, USA). Equal amounts of protein samples $(\sim 25 \mu \mathrm{g})$ were fractionated by SDS-PAGE (12\% polyacrylamide gels) and electrophoretically transferred to PVDF membrane (Millipore, Bedford, MA) using a Mini Trans-blot (Bio-Rad Laboratories, Shanghai). The membranes were blocked for $1 \mathrm{~h}$ in defatted milk (10\% in Tris-buffered saline with Tween-20 buffer), and incubated with primary antibodies $(1: 1200)$ at $4^{\circ} \mathrm{C}$ overnight. The primary antibodies included rabbit polyclonal anti-PPAR $\gamma 2$, anti-Osf2/Cbfa1, anti-GRP78, anti-GRP94, anti-ATF4, anti-CHOP, and anti-TNF- $\alpha$, all purchased from Santa Cruz Biotech (CA, USA). Next day, the membrane was incubated with horseradish peroxidase-conjugated secondary antibody (1:1000; Molecular Probes) diluted in PBS for $2 \mathrm{~h}$ at RT. Finally, the membrane was rinsed with PBS before scanning using the Infrared Imaging System (LI-COR Biosciences). $\beta$-actin was used as an internal control for equal input of protein samples, using anti- $\beta$-actin antibody. Western blot bands were quantified using the LI-COR ${ }^{\circledR}$-Image Studio Lite software by measuring the band intensity (Area $\times$ OD) for each group and normalizing to $\beta$-actin. The final results are expressed as fold changes by normalizing the data to the control values.

\section{Data analysis}

Results are presented as mean \pm S.E.M. Comparisons between the control and alcohol groups were conducted using unpaired Student $t$-test. A $P$ value of less than 0.05 was considered significant.

\section{Results}

Characterization of BMSCS

To ensure the purity of BMSCs in our cell preparation, we first determined the expression of CD34, CD105, and CD166 in the BMSCs by flow cytometry using the monoclonal antibodies CD34-FITC, CD105-PE, and CD166-PE in the undifferentiated states. We found that the BMSCs consistently expressed BMSC markers CD105 and CD166, but did not express hematopoietic 
Fig. 1. Alcohol-induced adipogenic lineage of bone marrow-derived stem cells (BMSCs), as revealed by Oil red 0 staining of lipid vacuoles. Upper panels: Representative images of Oil red 0 staining (magnification $\times 200$ ), showing negative staining of control BMSCs and the presence of oil red droplets in cells treated with alcohol $(100 \mathrm{mM})$ for 24 days. Lower panel: averaged number of adipocytes per field. ${ }^{* * *} P<0.001$ Alcohol vs Control; Student $t$-test; $\mathrm{n}=4$.

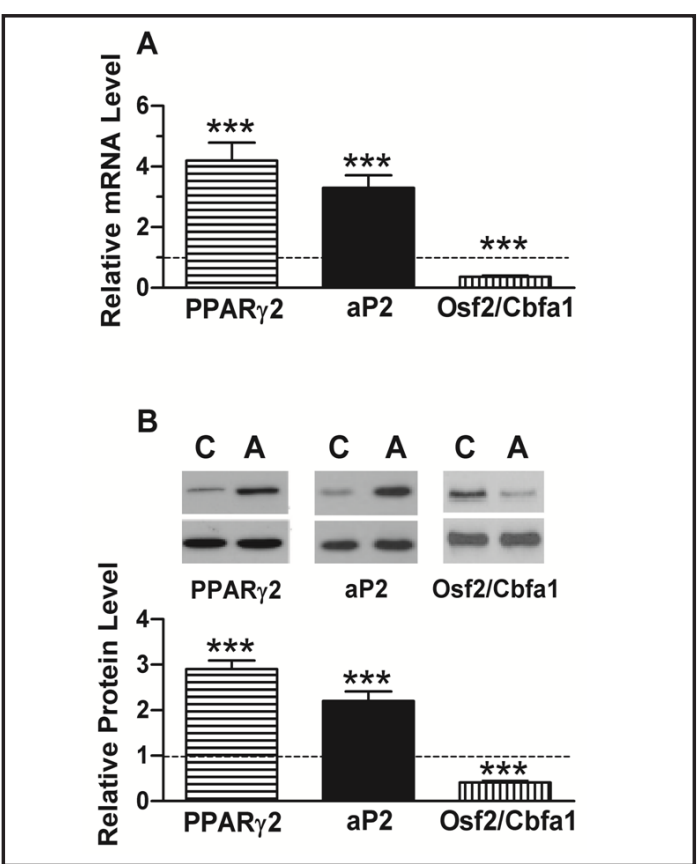

Fig. 2. Alcohol-induced upregulation of adipocyte markers PPAR 2 (70 kDa) and aP2 (15 kDa) and downregulation of osteoblast marker Osf2/Cbfa1 (55 kDa) at mRNA (A) and protein levels (B), determined by quantitative real-time RT-PCR (qPCR) and Western blot analysis in BMSCs. Shown are mean data in BMSCs treated with alcohol normalized to the values in untreated cells. Dash line indicates control values from untreated cells. C: the control group; A: the Alcohol group. ${ }^{* * *} P<0.001$ Alcohol vs Control; Student $t$-test; $\mathrm{n}=5$.
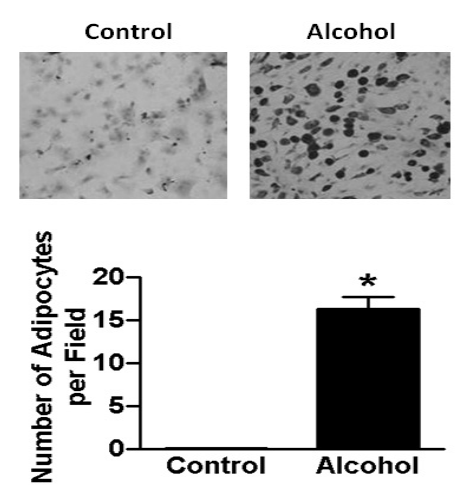

A Western Blot Analysis
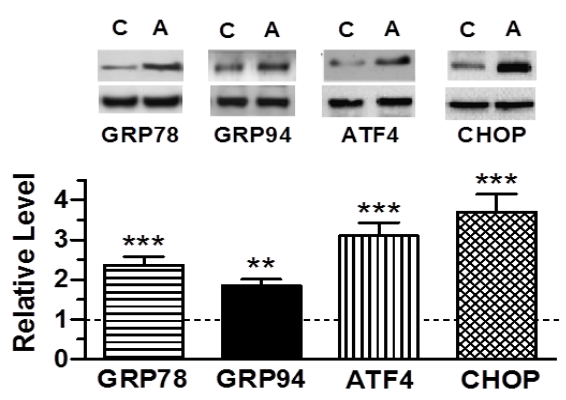

B

Caspase-3 Activity Assay

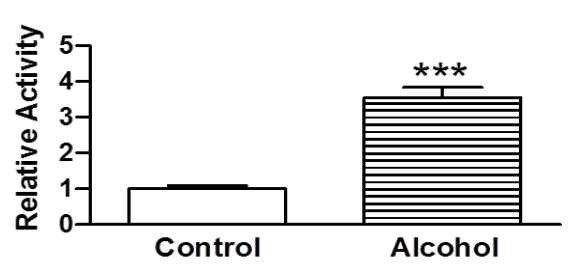

Fig. 3. Evidence for alcohol-induced endoplasmic reticulum (ER) stress in BMSCs. A, Upregulation of ER stress proteins glucose-response proteins GRP78 (78 kDa) and GRP94 (94 kDa), and transcription factors activating transcription factor 4 (ATF4; 38 $\mathrm{kDa}$ ) and C/EBP homologous protein (CHOP; $30 \mathrm{kDa}$ ) in BMSCs treated with alcohol (100 mM for 24 days), relative to untreated cells. Dash line indicates control values from untreated cells. B, Increased caspase 3 (an executioner of apoptosis) in BMSCs treated with alcohol. C: the control group; A: the Alcohol group. ${ }^{* *} P<0.01$ Alcohol vs Control; ${ }^{* * *} P<0.001$ Alcohol vs Control; Student $t$-test; $\mathrm{n}=4$.

cell marker CD34. The cells prepared by Percoll separation were composed of $99.1 \% \pm 7.3 \%$ of CD34 negative cells, $95.4 \% \pm 6.5 \%$ of CD105 positive cells and $93.3 \% \pm 5.7 \%$ of CD166 positive cells. 


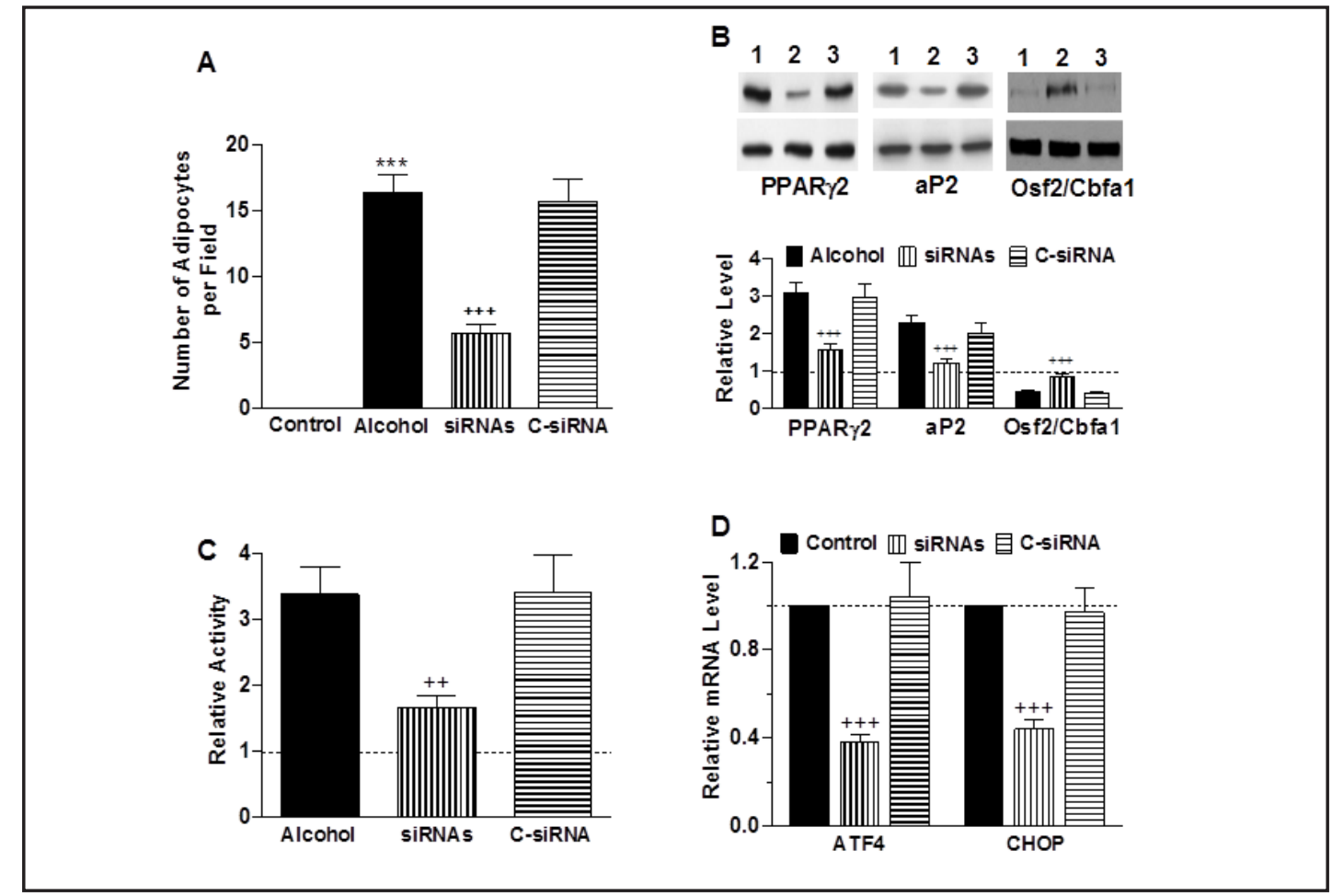

B

Fig. 4. Evidence for the involvement of ATF4 and CHOP thereby ER stress in alcohol-induced adipogenic lineage shift of BMSCs. A, Knockdown of ATF4 and CHOP by co-transfection of ATF4 siRNA and CHOP siRNA (siRNAs) reduced the increased number of adipocytes induced by alcohol (100 mM for 24 days). The negative control siRNA (C-siRNA) did not produce any significant effects. Control cells were mock-treated with lipofectamine2000. B, siRNAs to knockdown both ATF4 and CHOP diminished the increased adipocytes markers PPAR $\gamma 2$ and aP2 protein levels, and the decreased osteoblast marker OSf2/Cbfa1 protein level, induced by alcohol. C, siRNAs reduced the enhanced caspase 3 activity induced by alcohol. D, Verification of the efficacy of the siRNAs to knockdown both ATF4 and CHOP mRNAs and validation of C-siRNA as a negative control construct. 1: the Alcohol group; 2: Alcohol + siRNAs (co-transfection with ATF4 and CHOP siRNAs); 3: Alcohol + C-siRNA (negative control siRNA). ${ }^{* * *} P<0.001$ Alcohol vs Control; ${ }^{++} P<0.01$ siRNAs vs Alcohol alone; ${ }^{++} P<0.001$ siRNAs vs Alcohol alone; Student $t$-test; $\mathrm{n}=4$.

\section{Alcohol-induced adipogenic lineage of BMSCs}

To reproduce the long-recognized shift of BMSCs towards adipogenic lineage after chronic alcohol exposure under our experimental conditions, we conducted the following experiments. First, we consistently observed the accumulation of lipid droplets as visualised by Oil red 0 staining in cells treated with alcohol (Fig. 1), but not in the control group. Adipocyte quantification showed that the average lipid-positive cell number per field was $16.3 \pm 1.4$ in the alcohol group $(P<0.001)$.

Quantitative analysis of the mRNA levels by real-time RT-PCR (qPCR) revealed that the PPAR $\gamma 2$ and aP2 mRNAs in the alcohol group were significantly upregulated compared to the control group $(P<0.001$; Fig. $2 \mathrm{~A})$. In contrast, $0 \mathrm{sf} 2 / \mathrm{Cbfa} 1$, a marker gene for osteogenesis was down-regulated by $48.6 \%$ in the cells treated with alcohol compared to the control cells $(P<0.001$; Fig. 2B).

Similar results were obtained from Western blot analysis. The protein levels of PPAR $\gamma 2$ and aP2 were significantly increased in the alcohol group compared with the control group $(P<0.001$; Fig. 2C). On the other hand, the protein level of Osf2/Cbfa1 was markedly downregulated in BMSCs treated with alcohol compared with non-treated control cells $(P<0.01$; Fig. 2D). 
Fig. 5. Evidence for tumor necrosis factor-alpha $(\mathrm{TNF}-\alpha)$ as a possible downstream mediator of ATF4 and CHOP for adipogenic lineage shift of BMSCs. A, ATF4 and CHOP siRNAs downregulated the increased TNF- $\alpha$ mRNA level induced by alcohol. $B$, siRNAs downregulated the increased TNF- $\alpha$ protein level induced by alcohol. C-siRNA (negative control siRNA) did not produce any effects. Alcohol: the Alcohol group; siRNAs: Alcohol + siRNAs (cotransfection with ATF4 and CHOP siRNAs); C-siRNA: Alcohol + C-siRNA (negative control siRNA). ${ }^{* * *} P<0.001$ Alcohol vs Control; ${ }^{++} P<0.001$ siRNAs vs Alcohol alone; Student $t$-test; $\mathrm{n}=6$.

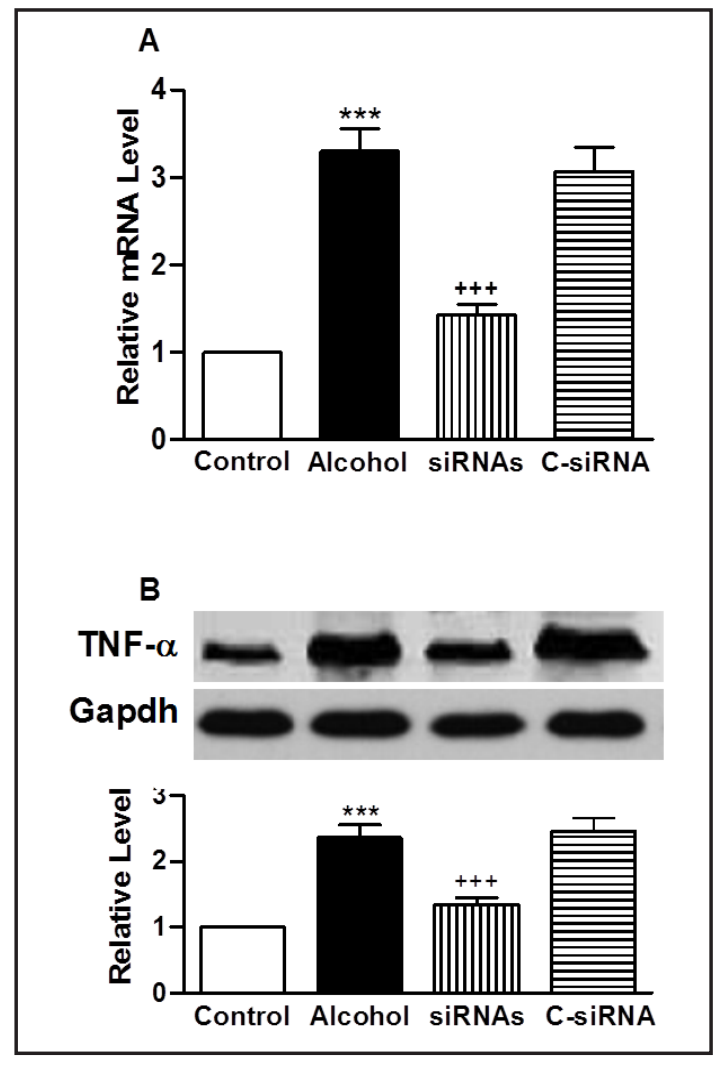

ER stress with chronic alcohol exposure

Immunoblotting analysis unambiguously showed robust upregulation of ER stress proteins GRP78 and GRP94 [36, 37], as well as their downstream mediators ATF4 and CHOP, in alcohol-treated BMSCs, as depicted in Figure 3. Additionally, caspase 3 activity was considerably enhanced in the alcohol group relative to the control cells (increased by approximately 3.5 fold; $P<0.01$ ). These results indicated that involvement of ER stress in BMSCs after prolonged exposure to alcohol.

Role of ER stress proteins ATF4 and CHOP in alcohol-induced adipogenic lineage of BMSCS

Differentiation of osteoblasts results from a complex molecular interplay involving the action of various transcription factors including ATF4 and CHOP [23-28]. Questions that came to our mind were whether the upregulation of these two ER stress proteins plays any role in alcohol-induced adipogenic lineage of BMSCs, and if yes, how it works. To answer these questions, we investigated the effects of simultaneous knockdown of ATF4 and CHOP by co-transfecting ATF4 and CHOP siRNAs. As illustrated in Figure 4, the siRNAs produced several alterations in BMSCs. First, the siRNAs reduced the number of lipid-positive cells per field from $16.3 \pm 1.4$ to $5.7 \pm 0.7$ in the presence of alcohol (Fig. 4A). Second, the siRNAs normalized the upregulated PPAR $\gamma 2$ and aP2 (Fig. 4B) and downregulated Osf2/Cbfa1 induced by ethanol (Fig. 4B). Third, the siRNAs mitigated the ethanol-induced increase in caspase 3 activity (Fig. 4C). The ability of the siRNAs to knockdown ATF4 and CHOP was verified by qPCR (Fig. 4D). The control siRNA did not cause any of these abovementioned changes.

Role of the TNF- $\alpha$ signaling axis in mediating the adipogenic effects of ATF4 and CHOP

High levels of TNF- $\alpha$ are known to inhibit osteoblastogenesis in culture and in vivo $[38,39]$; nevertheless, low doses can enhance osteoblast proliferation in culture [40]. It has also been reported that the anti-osteogenic effects of ethanol are at least partially mediated by the TNF- $\alpha$ signaling axis $[29,41]$. We wanted to elucidate whether the anti-osteogenic 


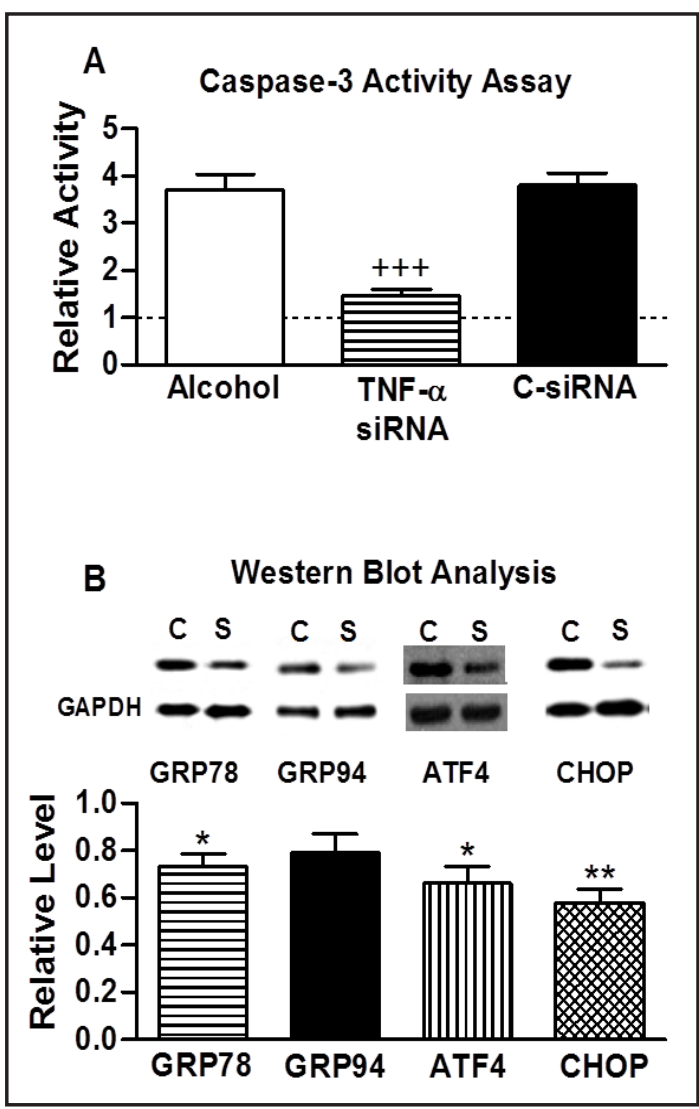

Fig. 6. Effects of TNF- $\alpha$ knockdown on ER stress. A, Knockdown of TNF- $\alpha$ by siRNA inhibits enhanced caspase 3 activity induced by alcohol exposure in BMSCs. Dash line indicates the level in untreated cells. ${ }^{++} P<0.001$ Alcohol+TNF- $\alpha$ siRNA vs Alcohol alone; Student $t$-test; $\mathrm{n}=5$. B, Downregulation of ER stress proteins glucose-response proteins GRP78 (78 kDa) and GRP94 (94 kDa), and transcription factors activating transcription factor 4 (ATF4; 38 $\mathrm{kDa}$ ) and C/EBP homologous protein (CHOP; 30 $\mathrm{kDa}$ ) in BMSCs treated with TNF- $\alpha$ siRNA in the presence of alcohol (100 mM for 24 days), relative to siRNA-nontreated cells. ${ }^{*} P<0.05$ and ${ }^{* *} P<0.01$ Alcohol+TNF- $\alpha$ siRNA vs Alcohol alone; Student $t$-test; $\mathrm{n}=3$.

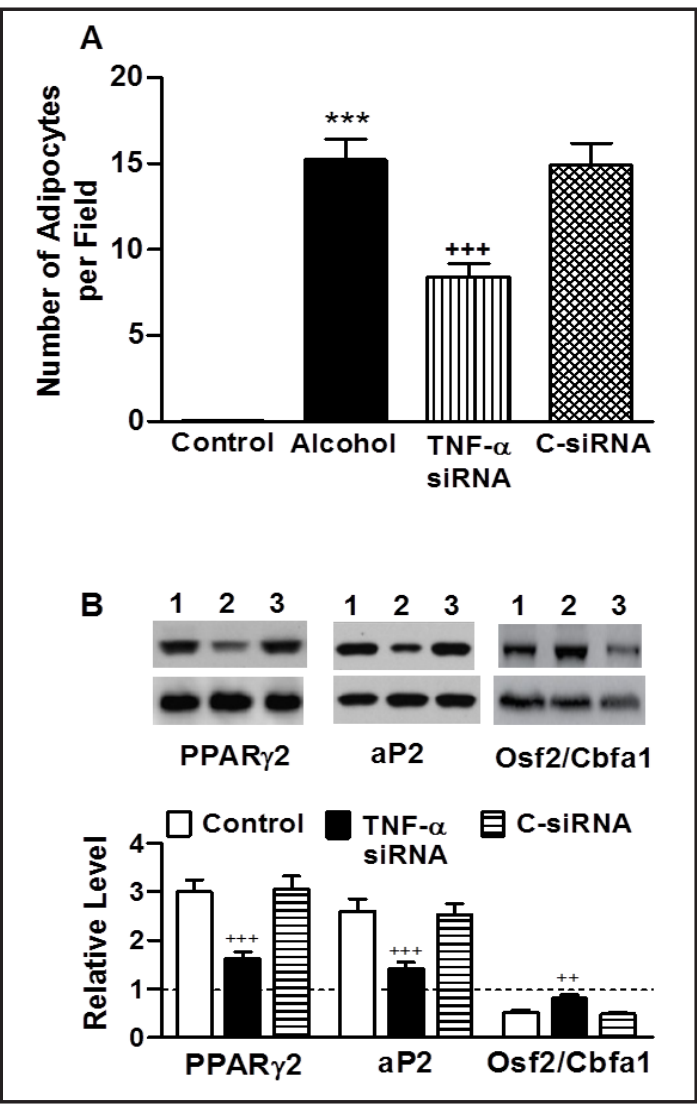

Fig. 7. Abrogation of alcohol-induced adipogenic lineage shift of BMSCs by TNF- $\alpha$ knockdown. A, TNF- $\alpha$ siRNA reduced the number of adipocytes. B, TNF- $\alpha$ siRNA decreased expression of PPAR $\gamma 2$ and aP2, and increased the expression of 0 sf $2 / \mathrm{Cbfa} 1$ in BMSCs treated with ethanol. C-siRNA: negative control siRNA. ${ }^{* * *} P<0.001$ Alcohol vs Control; ${ }^{++} P<0.01$ siRNA vs Alcohol alone; ${ }^{++} P<0.001$ siRNA vs Alcohol alone; Student $t$-test; $\mathrm{n}=5$.

properties of TNF- $\alpha$ and ATF4/CHOP are interrelated. To this end, we looked at the effects of ATF4/CHOP knockdown on TNF- $\alpha$ expression. As shown in Figure 5A \& 5B, ATF4/CHOP siRNAs substantially reduced the TNF- $\alpha$ mRNA and protein levels. To exclude the possibility that the observed effect was due to non-specific effects of the siRNA on TNF- $\alpha$, we also assessed the effects of alcohol exposure on TNF- $\alpha$ expression. The data presented in Figure $5 \mathrm{~B}$ clearly demonstrated remarkable increases of TNF- $\alpha$ at both mRNA and protein levels in cells treated with alcohol. More importantly, TNF- $\alpha$ siRNA ameliorated the ethanol-induced enhancement of caspase 3 activity (Fig. 6A). Meanwhile, knockdown of TNF- $\alpha$ also decreased the levels of ER stress markers GRP78 and GRP94, and consequently of ATF4 and CHOP proteins (Fig. 6B). Furthermore, TNF- $\alpha$ siRNA reduced the number of adipocytes (Fig. 7A), 
decreased expression of PPAR $\gamma 2$ and aP2 (Fig. 7B), and increased the expression of Osf2/ Cbfa1 in BMSCs treated with ethanol (Fig. 7B).

\section{Discussion}

Differentiation of osteoblasts results from a complex molecular interplay involving the action of various factors and signaling pathways, and deeper understanding of the mechanisms for bone formation and homeostasis is crucial for the management of bone disorders such as osteoporosis. While it has long been known that excessive alcohol consumption promotes adipogenesis and inhibits osteogenesis [5-9] and induces ER stress [11-13] that mediates alcoholic damages to many organs, the relationship between ER stress and osteogenesis disruption in BMSCs remained unknown. The present study provides evidence for the role of ER stress in mediating osteogenesis disruption after chronic alcohol exposure. While it is known that ethanol increases expression of TNF- $\alpha$ [30] and this upregulation may partly mediate the inhibitory effects of ethanol on osteogenesis disruption $[29,41]$, it remained unknown how TNF- $\alpha$ signaling is activated by ethanol in BMSCs. Our study generated data indicating the role of ER stress in activating TNF- $\alpha$. Moreover, our results also suggested that ATF 4 and CHOP mediate the ER stress-induced activation of TNF- $\alpha$ signaling leading to activation of caspase 3 or induction of BMSC apoptosis and reduced osteogenesis. These data also represent the first to reveal the osteogenesis-inhibitory actions of ATF4 and CHOP after alcohol exposure. These findings allow us to conclude that chronic alcohol exposure causes ER stress that in turn upregulates/activates ATF4 and CHOP that further activate TNF- $\alpha$. We therefore proposed the following signaling pathway for alcohol-induced destruction of osteogenesis: Ethanol / ER stress $\uparrow \uparrow \uparrow \rightarrow$ ATF4 \& CHOP $\uparrow \uparrow \rightarrow$ TNF- $\alpha \uparrow \uparrow \uparrow \rightarrow$ Osteoblasts $\downarrow \downarrow \downarrow$. It should be noted that ethanol has extensive effects on cellular signaling pathways and the signaling pathway proposed here likely represents merely a part of the mechanisms for the anti-osteogenic effects of ethanol.

ER stress is involved in a variety of pathological processes. However, it was not known whether it also takes a part in osteogenesis suppression in the presence of ethanol. It has been documented that excessive alcohol exposure induces ER stress [11-13]. The present study has provided a several lines of evidence for the presence of ER stress in response to ethanol treatment: upregulation of ER stress proteins GRP78 and GRP94, upregulation of transcription factors ATF4 and CHOP, and enhancement of caspase 3 activity. This ER stress activates TNF- $\alpha$ signaling by upregulating TNF- $\alpha$ at both mRNA and protein levels through ATF4 and CHOP, as simultaneous gene silencing of ATF4 and CHOP prevented upregulation of TNF- $\alpha$. Consistent with this result, knockdown of ATF4/CHOP repressed the expression of TNF- $\alpha$ at both mRNA and protein levels. Moreover, we identified several putative cis-acting elements for ATF4 and CHOP, respectively, within $2 \mathrm{~kb}$ upstream the TNF- $\alpha$ gene using the online transcription factor database Genomatix (http://www.genomatix.de/online_help/ help_matinspector/matinspector_help.html). Inhibition of either ATF4 and CHOP or TNF- $\alpha$ was able to alleviate ethanol-induced promotion of adipogenesis of BMSCs. These findings link ER stress to alcohol-induced adipogenic lineage of BMSCs. Notably, our finding that TNF- $\alpha$ siRNA caused reduction of GRP78 and GRP94 indicates that TNF- $\alpha$ can also function upstream of ER stress. It is conceivable that alcohol activates, but not directly upregulates, TNF- $\alpha$ which in turn contributes to ER stress induction. Then, activation of ATF4 and CHOP during ER stress upregulates TNF- $\alpha$ expression which can supposedly further exaggerate ER stress. If this is true, then there might be a positive feedback loop between alcohol and adipogenic lineage: ethanol $\rightarrow$ TNF- $\alpha$ activation $\uparrow \uparrow \rightarrow$ ER stress $\uparrow \uparrow \rightarrow$ ATF4/CHOP activities/ expression $\uparrow \uparrow \uparrow \rightarrow$ TNF- $\alpha$ expression $\uparrow \uparrow \uparrow \rightarrow$ adipogenic lineage shift $\uparrow \uparrow \uparrow$. This notion merits future studies to verify.

Intriguingly, despite that normal expression levels and activities of ATF4, CHOP and TNF- $\alpha$ are required for bone formation and homeostasis [23-25, 26-28, 40], excessive 
TNF- $\alpha$ has been shown to suppress osteogenesis [38, 39] and our results showed that aberrant upregulation of ATF4 and CHOP also caused a shift towards the adipogenic lineage. Knockdown of expression of either ATF4/CHOP or TNF- $\alpha$ produced a similar extent of rescue of osteogenesis in the presence of ethanol, indicating that ATF4/CHOP and TNF- $\alpha$ are in the same signaling pathway. These data support that the anti-osteogenic action of ATF4/ CHOP is mediated by TNF- $\alpha$. Moreover, these data explain why ATF4 and CHOP which are normally required for osteogenic shift of lineage enter into adipogenic remodeling when overexpressed during ER stress. Furthermore, these results also suggest that expression of ATF4/CHOP and TNF- $\alpha$ must be maintained within a certain level to maintain bone homeostasis; excessive downregulation or upregulation can disrupt the balance and cause abnormalities of bone development and physiology.

In conclusion, our data from the present study revealed a role of ER stress and ATF4/ CHOP in the ethanol-induced inhibition of osteogenesis, and activation of TNF- $\alpha$ signaling by ATF4/CHOP links ER stress to adipogenic lineage in response to alcohol stimulation. Thus, ATF4, CHOP, and TNF- $\alpha$ may be critical for both bone formation and maintenance. These findings help us better understand the mechanisms for the osteogenesis and adipogenesis of BMSCs.

\section{Sources of Funding}

This study was supported partially by Guangxi Natural Science Foundation of China (Grant \# 2010GXNSFA013223), and by the key topics of the Guangxi Health Department (Grant \# 2010096).

\section{Conflicts of Interest}

The authors declare no conflicts of interest for this study.

\section{References}

1 Włodarski KH: Haematopoietic and osteogenic bone marrow stem cells. Ortop Traumatol Rehabil 2011;13:439-447.

2 Pignolo RJ, Shore EM: Circulating osteogenic precursor cells. Crit Rev Eukaryot Gene Expr 2010;20:171180.

-3 Wu JY, Scadden DT, Kronenberg HM: Role of the osteoblast lineage in the bone marrow hematopoietic niches. J Bone Miner Res 2009;24:759-764.

4 Heino TJ, Hentunen TA: Differentiation of osteoblasts and osteocytes from mesenchymal stem cells. Curr Stem Cell Res Ther 2008;3:131-145.

5 Wahl EC, Aronson J, Liu L, Liu Z, Perrien DS, Skinner RA, Badger TM, Ronis MJ, Lumpkin CK Jr: Chronic ethanol exposure inhibits distraction osteogenesis in a mouse model: role of the TNF signaling axis. Toxicol Appl Pharmacol 2007;220:302-310.

6 Wang Y, Li Y, Mao K, Li J, Cui Q, Wang GJ: Alcohol-induced adipogenesis in bone and marrow: a possible mechanism for osteonecrosis. Clin Orthop Relat Res 2003;410:213-224.

-7 Cui Q, Wang Y, Saleh KJ, Wang GJ, Balian G: Alcohol-induced adipogenesis in a cloned bone-marrow stem cell. J Bone Joint Surg Am 2006;88 Suppl 3:148-154.

8 Rosa ML, Beloti MM, Prando N, Queiroz RH, de Oliveira PT, Rosa AL: Chronic ethanol intake inhibits in vitro osteogenesis induced by osteoblasts differentiated from stem cells. J Appl Toxicol 2008;28:205-211.

9 Diamond T, Stiel D, Lunzer M, Wilkinson M, Posen S: Ethanol reduces bone formation and may cause osteoporosis. Am J Med 1989;86:282-288. 
Chen et al.: TNF- $\alpha$ in Alcohol-Induced Adipogenic Lineage of MSC

10 Purohit V: Introduction to the alcohol and osteoporosis symposium. Alc Clin Exp Res 1997;21:383-384.

11 Chen JR, Lazarenko OP, Shankar K, Blackburn ML, Badger TM, Ronis MJ: A role for ethanol-induced oxidative stress in controlling lineage commitment of mesenchymal stromal cells through inhibition of Wnt/beta-catenin signaling. J Bone Miner Res 2010;25:1117-1127.

12 Nguyen KH, Lee JH, Nyomba BL: Ethanol causes endoplasmic reticulum stress and impairment of insulin secretion in pancreatic $\beta$-cells. Alcohol 2012;46:89-99.

13 Nishitani Y, Matsumoto H: Ethanol rapidly causes activation of JNK associated with ER stress under inhibition of ADH. FEBS Lett 2006;580:9-14.

14 Wek RC, Jiang HY, Anthony TG: Coping with stress: eIF2 kinases and translational control. Biochem Soc Trans 2006;34:7-11.

15 Vattem KM, Wek RC: Reinitation involving upstream ORFs regulates ATF4 mRNA translation in mammalian cells. Proc Natl Acad Sci USA 2004;101:11269-11274.

-16 Saito A, Ochiai K, Kondo S, Tsumagari K, Murakami T, Cavener DR, Imaizumi K: Endoplasmic reticulum stress response mediated by the PERK-eIF2(alpha)-ATF4 pathway is involved in osteoblast differentiation induced by BMP2. J Biol Chem 2011;286:4809-4818.

17 Karpinski BA, Morle GD, Huggenvik J, Uhler MD, Leiden JM: Molecular cloning of human CREB-2: an ATF/ CREB transcription factor that can negatively regulate transcription from the cAMP response element. Proc Natl Acad Sci USA 1992;89:4820-4824.

18 Marciniak SJ, Yun CY, Oyadomari S, Novoa I, Zhang Y, Jungreis R, Nagata K, Harding HP, Ron D: CHOP induces death by promoting protein synthesis and oxidation in the stressed endoplasmic reticulum. Genes \& Development 2006;18:3066-3077.

19 Oyadomari S, Mori M: Roles of CHOP/GADD153 in endoplasmic reticulum stress. Cell Death Differ 2004;11:381-389.

-20 Galehdar Z, Swan P, Fuerth B, Callaghan SM, Park DS, Cregan SP: Neuronal apoptosis induced by endoplasmic reticulum stress is regulated by ATF4-CHOP-mediated induction of the Bcl-2 homology 3-only member PUMA. J Neurosci 2010;30:16938-16948.

-21 Nishitoh H: CHOP is a multifunctional transcription factor in the ER stress response. J Biochem 2012;151:217-219.

-22 Hamamura K, Yokota H: Stress to endoplasmic reticulum of mouse osteoblasts induces apoptosis and transcriptional activation for bone remodeling. FEBS Lett 2007;581:1769-1774.

23 Yang X, Matsuda K, Bialek P, Jacquot S, Masuoka HC, Schinke T, Li L, Brancorsini S, Sassone-Corsi P, Townes TM, Hanauer A, Karsenty G: ATF4 is a substrate of RSK2 and essential regulator of osteoblast biology: Implication for Coffin-Lowry Syndrome. Cell 2004;117:387-398.

24 Komori T: Regulation of osteoblast differentiation by transcription factors. J Cell Biochem 2006;99:12331239.

-25 Franceschi RT, Ge C, Xiao G, Roca H, Jiang D: Transcriptional regulation of osteoblasts. Ann N Y Acad Sci 2007;1116:196-207.

-26 Pereira RC, Stadmeyer LE, Smith DL, Rydziel S, Canalis E: CCAAT/Enhancer binding protein homologous protein (CHOP) decreases bone formation and causes osteopenia. Bone 2007;40:619-626.

-27 Shirakawa K, Maeda S, Gotoh T, Hayashi M, Shinomiya K, Ehata S, Nishimura R, Mori M, Onozaki K, Hayashi H, Uematsu S, Akira S, Ogata E, Miyazono K, Imamura T: CCAAT/Enhancer binding protein homologous protein (CHOP) regulates osteoblasts differentiation. Mol Cell Biol 2006;26:6105-6116.

28 Kveiborg M, Sabatakos G, Chiusaroli R, Wu M, Philbrick WM, Horne WC, Baron R: DeltaFosB induces osteosclerosis and decreases adipogenesis by two independent cell-autonomous mechanisms. Mol Cell Biol 2004;24:2820-2830.

-29 Wahl EC, Perrien DS, Aronson J, Liu Z, Fletcher TW, Skinner RA, Feige U, Suva LJ, Badger TM, Lumpkin CK Jr: Ethanol-induced inhibition of bone formation in a rat model of distraction osteogenesis: a role for the tumor necrosis factor signaling axis. Alcohol Clin Exp Res 2005;29:1466-1472.

30 Perrien DS, Liu Z, Wahl EC, Bunn RC, Skinner RA, Aronson J, Fowlkes J, Badger TM, Lumpkin CK Jr: Chronic ethanol exposure is associated with a local increase in TNF-alpha and decreased proliferation in the rat distraction gap. Cytokine 2003;23:179-189.

-31 Huang Q Zhang H, Pei FX, Chen ZY, Wang GL, Shen B, Yang J, Zhou ZK, Kong QQ: Use of small interfering ribonucleic acids to inhibit the adipogenic effect of alcohol on human bone marrow-derived mesenchymal cells. Int Orthop 2010;34:1059-1068. 
Chen et al.: TNF- $\alpha$ in Alcohol-Induced Adipogenic Lineage of MSC

32 Pittenger MF, Mackay AM, Beck SC, Jaiswal RK, Douglas R, Mosca JD, Moorman MA, Simonetti DW, Craig S, Marshak DR: Multilineage potential of adult human mesenchymal stem cells. Science 1999;284:143-147.

-33 Klein RF: Alcohol-induced bone disease: impact of ethanol on osteoblast proliferation. Alcohol Clin Exp Res 1997;21:392-399.

34 Komatsu S, Miyazawa K, Moriya S, Takase A, Naito M, Inazu M, Kohno N, Itoh M, Tomoda A: Clarithromycin enhances bortezomib-induced cytotoxicity via endoplasmic reticulum stress-mediated CHOP (GADD153) induction and autophagy in breast cancer cells. Int J Oncol 2012;40:1029-1039.

-35 Rzymski T, Milani M, Pike L, Buffa F, Mellor HR, Winchester L, Pires I, Hammond E, Ragoussis I, Harris AL: Regulation of autophagy by ATF4 in response to severe hypoxia. Oncogene 2010;29:4424-4435

36 Liu ES, Lee AS: Common sets of nuclear factors binding to the conserved promoter sequence motif of two coordinately regulated ER protein genes, GRP78 and GRP94. Nucleic Acids Res 1991;19:5425-5431.

37 Argon Y, Simen BB: GRP94, an ER chaperone with protein and peptide binding properties. Semin Cell Dev Biol 1999;10:495-505.

-38 Nanes MS: Tumor necrosis factor- $\alpha$ : molecular and cellular mechanisms in skeletal pathology. Gene 2003;321:1-15.

-39 Frost A, Jonsson K, Nilsson 0, Ljunggren 0: Inflammatory cytokines regulate proliferation of cultured human osteoblasts. Acta Ortho Scand 1997;68:91-96.

40 Gerstenfeld L, Cho T, Kon T, Aizawa T, Cruceta J, Graves B, Einhorn T: Impaired intramembranous bone formation during bone repair in the absence of tumor necrosis factor-alpha signaling. Cells Tissues Organs 2001;169:285-294.

\$1 Perrien DS, Brown EC, Fletcher TW, Irby DJ, Aronson J, Gao GG, Skinner RA, Hogue WR, Feige U, Suva LJ, Ronis MJ, Badger TM, Lumpkin CK Jr: Interleukin-1 and tumor necrosis factor antagonists attenuate ethanolinduced inhibition of bone formation in a rat model of distraction osteogenesis. J Pharmacol Exp Ther 2002;303:904-908. 